\title{
Household Appliances Identification: An integrative workshop for the Electrical Engineering degree
}

\author{
Alvaro Gomez, Pablo Massaferro, Camilo Marino, Ignacio Irigaray, Andres Cardozo and Alicia Fernandez \\ Instituto de Ingenierıa Electrica, Facultad de Ingenieria - Universidad de la Republica \\ fagomez, pmassaferro, cmarino, irigaray, acardozo, aliciag@fing.edu.uy
}

\begin{abstract}
In the Electrical Engineering degree, the integration of theoretical and practical knowledge is essential. Throughout the degree, there must be instances where the students can face specific problems and test their learning. With this in mind, an integrative workshop is implemented in the third year that seeks to strengthen skills such as: analysis and design of signal conditioning circuits, designing and printing circuits, signal acquisition and processing, pattern recognition and classification, integration of a system. The problem of the identification of household appliances from consumption records presents multi-ple challenges suitable to develop all the desired skills and also for the initiation to research.
\end{abstract}

Index Terms_-Integrative workshop, Electrical Engineering degree, load identification 